\title{
The Receiving Process of Single Carrier Signal Based On Blind Equalization Technology
}

\author{
Tao $\mathrm{Xu}^{1, \mathrm{a}^{*}}$, Yu Feng ${ }^{1, \mathrm{~b}}$, Zhifeng Wang ${ }^{1, \mathrm{~b}}$, Zhihua $\mathrm{Hu}^{1, \mathrm{~b}}$ \\ ${ }^{1}$ Shanghai second polytechnic university, China \\ axutao@sspu.edu.cn, ${ }^{b}$ fengyu@ sspu.edu.cn
}

\begin{abstract}
Keywords: Single carrier; Mobile digital TV; Blind equalization; Decision guiding.
Abstract. In this paper, the equilibrium issue exists in the single carrier mobile digital TV signal processing is discussed first, and in order to improve the balance performance, how to make equalizer switch automatically between blind equalization algorithm and decision oriented algorithm is researched. Otherwise the architecture of adaptive switch between blind equalization mode and decision oriented mode based on single carrier receiving is also given. In this paper, the equalizer mode switching algorithm with double expected threshold is put forward, and the expected output error is used to control the switching between two equalizer modes. It guarantees the smooth switch of equalizer mode and reduces the convergence error. The simulation results show that with a suitable expected threshold, compared with predecessors of switching algorithm, the algorithm put forward in this paper can enhance the performance in some way.
\end{abstract}

\section{Introduction}

The mobile digital TV technology in America is based on the digital communication technology of single carrier 8 VSB modulation. Its difference with the common digital TV signal is that it can ensure the stability of receiving image information when transmitter and receiver are in relative motion. The time-varying characteristics of channel and Doppler frequency shift are the two most important factors of affecting the receiving quality. Therefore, opening the eye diagram quickly and providing effective video information for users are the prior problems needed to resolve. ATSC M/H agreement of America mobile digital TV provides a lot of training sequence to support the correction of receiving data. But for the single carrier signal receiver, equalizer convergence algorithm based on data recognition is simple, and easy to implement, it also can adapt the variation caused by slow channel fading. But this algorithm is just suitable for the case that equalizer eye diagram opened [1]. When training sequence and loop multiplexing data can't open the equalizer eye diagram or equalizer can't converge to the correct working point due to strong short-term channel fading, using LMS algorithms may cause the equalizer can't work and converge normally. At this time, the way of blind equalization is needed to improve the eye diagram opening degree of the equalizer output signal, in order to make the eye diagram open adaptively and make the equalizer converge again.

Due to causes such as channel multipath interference, the received signal distortion is serious. Under this situation, the receiver can recover data only by adaptive equalization with the received signal sequence itself, and it does not need training sequence. This method above is blind equalization. This method is an adaptive filtering scheme that is no need to provide signal expected response to receiver, but realize received signal equilibrium according to the characteristics of signals. It is close to expected response in a sense. Due to the expected response of signal is unknown, so it is blind. But blind equalization algorithm can use a non-linear variation to estimate the expected response of produced signals. It balances the error between output results and criterion results, and using approximate rule to make the equalizer output close to or recover to the input signal. Compared with common equalizer, the application range and convergence domain of blind equalization is wide.

According to how the data nonlinear change, blind equalization can be divided into the following four categories $[2,3]$

(1) Bussgang algorithm

(2) The algorithm based on high order spectrum theory 
(3) Blind estimation based on the input sequence

(4) Blind equalization method based on neural network theory

Simplified model of blind equalization system is shown in Fig.1 below.

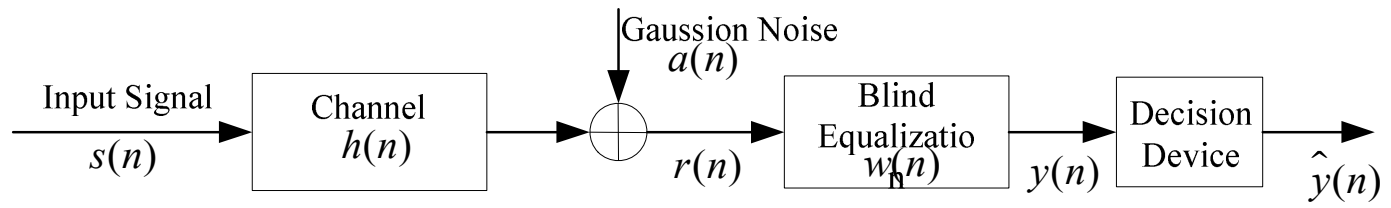

Fig. 1. Simplified model of blind equalization system

In the Fig. 1 above, $s(n)$ is input signal sequence, $h(n)$ is the multipath dispersion impulse response function of communication channel, $a(n)$ is Gaussian noise sequence be added to the channel, $r(n)$ is data sequences after input signal through multipath interference, namely it is the observed signals of equalizer. $w(n)$ is the function of blind Equalizer tap, $y(n)$ is the output signal sequence after equilibrium, $\hat{y}(n)$ is output sequence after judgement. Thereinto, the input signal $r(n)$ of blind equalizer can be the result that the convolution of channel function and sending data added Gaussian noise. Its equation is

$$
r(n)=\sum_{i=-\infty}^{\infty} w(i) s(n-i)+a(n)
$$

For blind equalization, although it can realize adaptive data equilibrium according to statistical characteristics of sending signal, especially it can accelerate the convergence of equalizer under the condition that channel characteristic is very bad. Compared with the decision guiding algorithm, ordinary blind equalization algorithm still exists residual error after converging. Therefore, in order to accelerate the convergence speed of equalizer and reduce the system residual error, after equalizer output adjusted by blind equalization meets the convergence conditions, it is need to switch blind equalization model to guide decision model. At the same time, in order to prevent the decision oriented equilibrium model from error adjustment under the condition of output without convergence, blind equalization model need switch automatically when equalizer output error arrives a certain degree.

\section{Algorithm Analysis}

In this section firstly several common switching algorithms are introduced, then on the basis of them a new switching algorithm is proposed, in an effort to accelerate the convergence of equalizer and guarantee the work stability of the equalizer.

BG Algorithm. Based on traditional Sato algorithm and decision oriented algorithm, Benveniste.et.al combined the two together, and proposed the advantages of them as well as BG algorithm [4].

As the above section proposed, the error of Sato algorithm is $e(n)=\alpha \operatorname{sgn}[y(n)]-y(n)$, thereinto $y(n)$ is the output result of equalizer, $\alpha=E\left[s^{2}(n)\right] / E[|s(n)|]$. The algorithm error calculation formula shows that error and zero still have more differences even after the equalizer converging. That is to say, there is still existing larger excessive error, unless the input signal $s(n)$ is $\pm \alpha$. But for decision oriented algorithm, its judgment error is $e(n)=\tilde{y}(n)-y(n)$. When output error is big, the robustness of blind equalization algorithm is better than that of decision oriented algorithm, but convergence is not as good as that of decision oriented algorithm. Because the judgment error $e(n)$ of blind equalization algorithm can be equal or close to zero.

Combined traditional decision guiding algorithm with Sato algorithm, BG algorithm can be achieved, and the tap recursive equation is 


$$
W(n+1)=W(n)+\mu r^{*}(n) e_{B G}(n)
$$

Error signal is

$$
e_{B G}(n)=m_{1} e_{D D}(n)+m_{2}\left|e_{D D}(n)\right| e_{\text {Sato }}(n)
$$

Thereinto $m_{1}, m_{2}$ are constant, $e_{D D}(n)=\tilde{y}(n)-y(n), e_{\text {Sato }}(n)=\alpha \operatorname{sgn}[y(n)]-y(n)$.

When the signal is disturbed by multipath impulse in the period of transmission, equalizer output error $|e(n)|$ is larger, at this moment the second part of Eq. 3 plays a leading role, and equalizer switches to blind equalization model. On the other hand, the equalizer switches to decision model. However, BG algorithm reduces the equalizer residual error at the price of sacrificing the convergence speed.

GPEA-G Algorithm. Using the model that $p=2$ in Godard algorithm to replace the error of BG algorithm, GPEA - $\mathrm{G}$ algorithm can be achieved [5]. The tap recursive equation is

$$
W(n+1)=W(n)+\mu r *(n) e_{G G}(n)
$$

Error signal is

$$
e_{G G}(n)=m_{1} e_{D D}(n)+m_{2}\left|e_{D D}(n)\right| e_{C M A}(n)
$$

In which, $e_{D D}(n)=\tilde{y}(n)-y(n), e_{C M A}(n)=y(n)\left(R_{2}-|y(n)|^{2}\right)$.

The convergence characteristic of GPEA-G algorithm is similar to CMA algorithm, and residual characteristic is similar to that of decision algorithm. But when this algorithm reduces the residual error of equalizer, it also reduces the convergence speed of the equalizer at the same time.

The convergence speed of Godard algorithm is faster than that of Sato algorithm, but the residual error is larger than that of BG algorithm.

\section{Mode Switching Algorithm Proposed}

Proposed Algorithm. Aiming at the advantages and disadvantages of the previous two switching algorithm, a blind equalization and decision oriented mode switching algorithm is proposed in this paper, it keeps the stability of equalizer algorithm and at the same time it also makes the convergence speed faster. Here we call this algorithm DET - DMA.

Switch structure figure of DFE structure equilibrium mode based on ATSC Mobile DTV standards is shown in the following Fig. 2.

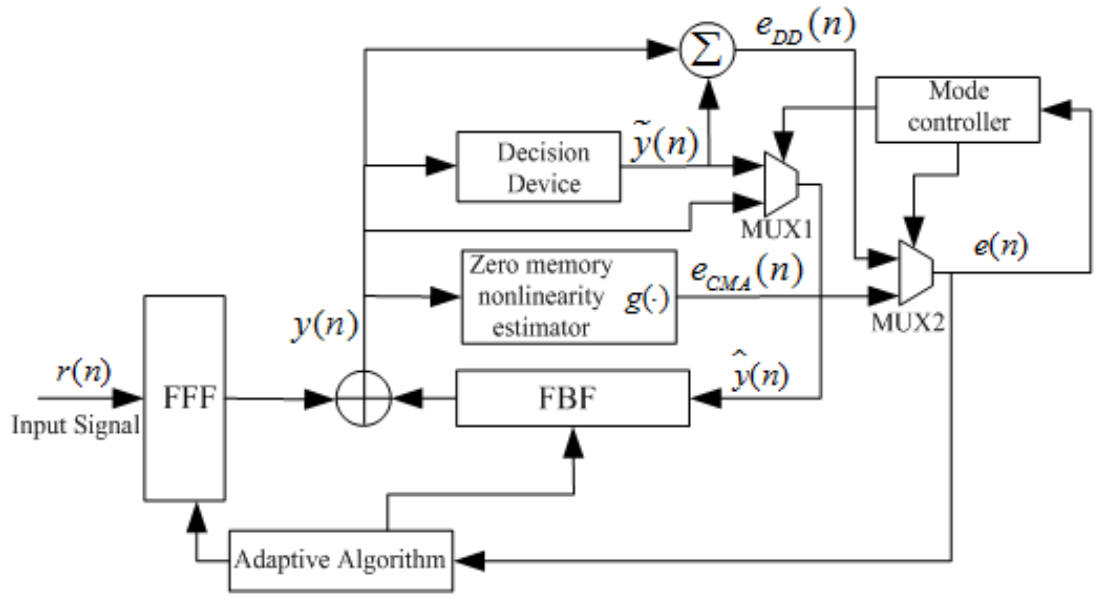

Fig. 2. Mode switch block diagram of blind equalization and decision guiding equalization

As Fig. 2 shown, the mode switch between blind equalization and decision guiding equalization is controlled by mode controller. After the input signal passes through decision feedback equalizer, then the output result is gained, and it is $y(n)$. MUX1 can control the data input to the feedback filter, it 
decides the data is the result $\hat{y}(n)$ after judgment or the result $y(n)$ directly after the equalizer. When the equalizer works in the decision guiding mode, the input of feedback filter is $\hat{y}(n)$ which is after decision. While the equalizer works in the blind equalization mode, the input of feedback filter is the result $y(n)$ after equalization. In the judgment mode, the error signal is $e_{D D}(n)$. In the blind equalization mode, the error signal is $e_{C M A}(n)$. MUX2 can decide which error signal controls the update of filter tap coefficients. According to the input error signal $e(n)$, mode controller determines the operation mode of the equalizer, and controls the switching between switch MUX1 and MUX2.

Update equations about forward and backward filter tap of equalizer based on LMS algorithm can be expressed as

$$
\left\{\begin{array}{l}
c_{i}[n+1]=c_{i}[n]-\mu e[n] r[n-i] \\
b_{j}[n+1]=b_{j}[n]+\mu e[n] \hat{y}[n-j]
\end{array}\right.
$$

Specific, the tap update algorithm of blind equalization model and decision orientation model can be expressed respectively as

$$
\begin{aligned}
& \left\{\begin{array}{l}
c_{i}[n+1]=c_{i}[n]-\mu_{D D} e_{D D}[n] r[n-i] \\
b_{j}[n+1]=b_{j}[n]+\mu_{D D} e_{D D}[n] \tilde{y}[n-j]
\end{array} \quad D D-\bmod e\right. \\
& \left\{\begin{array}{l}
c_{i}[n+1]=c_{i}[n]-\mu_{C M A} e_{C M A}[n] r[n-i] \\
b_{j}[n+1]=b_{j}[n]+\mu_{C M A} e_{C M A}[n] y[n-j]
\end{array} \quad C M A-\bmod e\right.
\end{aligned}
$$

In which

$$
\begin{cases}e(n)=e_{D D}(n)=\tilde{y}(n)-y(n) & D D-\bmod e \\ e(n)=e_{C M A}(n)=y(n)\left(R_{2}-|y(n)|^{2}\right) & C M A-\bmod e\end{cases}
$$

The $\mathrm{R}_{2}$ is as a constant here, it represents the expectation ratio of input symbols in the 8-VSB system.

$$
R_{2}=\frac{E\left\{|s(n)|^{4}\right\}}{E\left\{|s(n)|^{2}\right\}}=37
$$

Due to the step length of CMA algorithm is far less than that of decision oriented pattern, here we assumed that the relationship of the two is

$$
\mu_{C M A}(n)=10^{-2} \cdot \mu_{D D}(n)
$$

In the control process above, switching mode in which position is crucial. The appropriate switch can optimize the performance of the equalizer and avoid the error propagation. If switching blind equalization mode to decision oriented mode prematurely, it will cause the algorithm does not converge or there will be a large steady-state error after convergence. But if switching too late, it will lead to long time delay of the equalizer convergence process. Therefore, on the basis of the above discussion about switching algorithm, a equalizer mode switching algorithm based on double expectations threshold is proposed in this paper, the most important advantage of it is automatically switching rather than manual switching, and the switch control is determined by the expectations of equalizer output error level. Compared with other blind equalization algorithms, this algorithm can make smooth switching between the two modes, and sudden work disturb noise won't be caused. 
Mathematical expectation $E[\tilde{y}(n)-y(n)]$ of the difference between decision result and equilibrium output result is taken as equalizer mode switching parameters in this paper, and using two thresholds $T H_{d c}$ and $T H_{c d}$ to control the mutual switch between the two equilibrium models. In which, the $T H_{d c}$ controls the equilibrium model to switch from decision oriented to normal blind equalization mode. When output error expectancy of the equalizer exceeds the threshold, that is $E[\tilde{y}(n)-y(n)]>T H_{d c}$, mode controller controls the switching between switch MUX1 and MUX2, and the equalizer changed into blind equalization mode. $T H_{c d}$ is used to control the equalizer switching from blind equalization mode to decision oriented mode. When the input error expectancy of equalizer is less than the threshold, that is $E[\tilde{y}(n)-y(n)]<T H_{c d}$, mode controller makes MUX1 and MUX2 switching between error and feedback filter input, in order to make the equalizer get into decision oriented mode. Considering that the robustness of decision oriented mode is worse than that of blind equalization, but in the case of little error the decision oriented mode can accelerate the convergence of equalizer, and the output error formulas of the blind equalization model and decision orientation model are different, so we assumes $T H_{d c}>T H_{c d}$. It can be understood that only when the output error expectation of decision oriented mode exceeds $T H_{d c}$, the equalizer can switch to the blind equalization model. Only when the output error expectation of blind equalization mode is better than $T H_{c d}$, the equalizer can switch to the blind equalization model.

Simulation and the Result Analysis. Due to the selection of threshold simulation has enormous influence on the simulation performance of the system. In general, We assume $T H_{d c}=0.1$, $T H_{d c}-T H_{c d}=r$. For the communication system, the symbol error rate can reflect the performance index of equalizer better. In order to simulate the equalizer performance with different $r$ values, we simulate different $r$ values and the equalizer performance under different signal-to-noise ratio. Here the step length is set to be 0.005 , static Brazil C channel is used.

It can be seen from Fig. 3, when $r=0.005$, the performance is the worst. The performance while $\mathrm{r}=0.01$ is better. At the range from 0.015 to 0.025 , the performance gap is little. At the same time, considering that the greater the $r$ value is, the slower the equalizer convergence is, so in this paper $\mathrm{r}=0.015$ is chosen as the difference between the two. That is $T H_{c d}=0.085$.

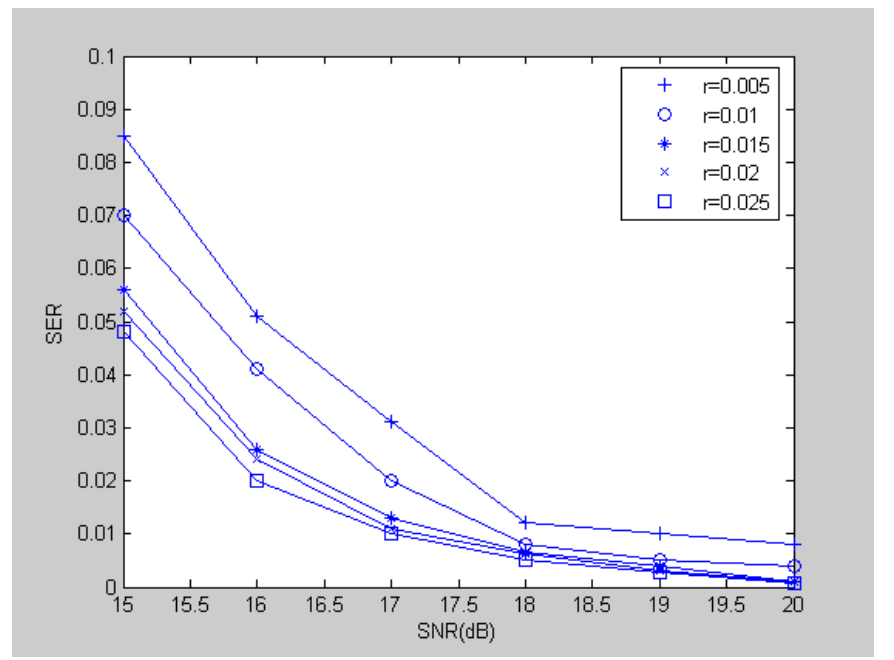

Fig. 3. The performance comparation with different $r$ values

To compare the performance difference between the proposed switching algorithm and the equalizer mode switching algorithm that discussed before, in this paper, simulation experiments are did respectively in the Brazil $\mathrm{C}$ channel and the B channel in ITU walking model. The simulation results are shown in Fig. 4 and Fig.5. 
Through the simulation we can see, compared with the GPEA-G algorithm, the proposed DET-DMA algorithm reduced the output error rate, and improved the output performance of the equalizer.

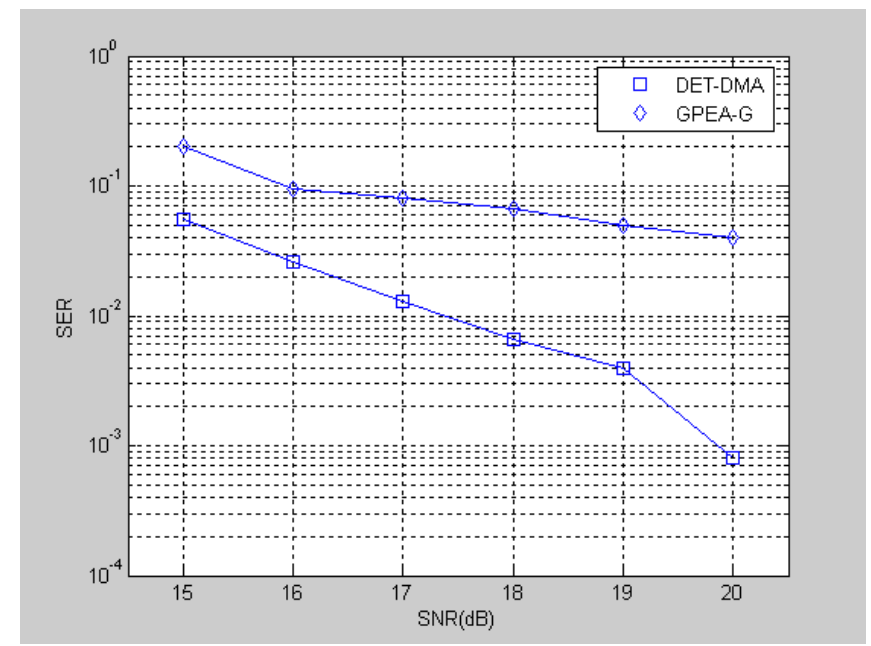

Fig. 4. Equalizer performance comparation under Brazil C channel

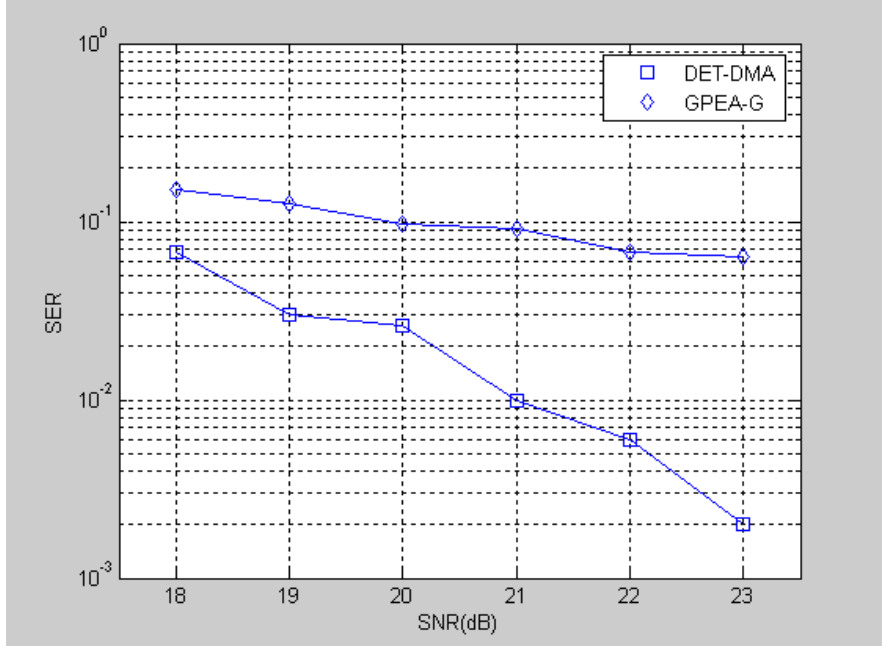

Fig. 5. Equalizer performance comparation under ITU-B channel

\section{Summary}

In this paper, the definition of the blind equalizer is given and equalization algorithms are classified first. Then a mathematical description of the blind equalizer is given. Starting form the Bussgang algorithm adopted in this paper, the basic principle of this algorithm is analyzed, and some special examples based on this algorithm are introduced. Otherwise, aimed at these examples, researches are done and performances are analyzed from the aspect of the theoretical derivation and the convergence characteristics.

Based on the previous theoretical basis of blind equalization, how to make the equalizer automatically switch between the blind equalization algorithm and decision directed algorithm is researched, and the equalization performance is improved. First the BG algorithm and GPED-G algorithm are introduced, and then the equalizer work structure about blind equalization and decision directed mode switching based on ATSC Mobile received DTV is presented. The mode switching algorithm with dual threshold is proposed, and output error expectation is adopted to control the switching between two equilibrium models. The dual threshold method proposed guaranteed the smooth switching of equalizer mode, prevented the generation of switching disturbance, and reduced the convergence error generated in accelerating convergence. Through the analysis of simulation results, when the desired threshold is selected appropriately, using this algorithm can make the performance have a certain improvement. 


\section{Acknowledgements}

This work was financially supported by the Shanghai Second Polytechnic University Key Discipline Construction( $4^{\text {th }}$ term)-Control Theory \& Control Engineering(No.XXKPY1038) .

\section{References}

[1] Jong-seob Baek, Won-kyu Paik, Yeon-woo Lee, Channel estimation-based equalizationtechnique for 8-VSB DTV system in multi-path channel, IEEE Conferences ICCE, 2005, 87-88.

[2] Hae-Sock Oh, Dong Seog Han, Chae-Hyun Lim, Sparse equalizer using adaptive weight activation for fast start-up in ATSC DTV systems, IEEE Conferences ICCE, 2006, Vol.52 (1) 92-96.

[3] J.M.Kim, Y.H.Lee, Fast Initialization of Equalizers for VSB-Based DTV Transceivers in Multipath Channel, IEEE Transactions on Broadcasting, 2005, Vol.51 (1) 133-138.

[4] Pascal Bianchi, Philippe Loubaton, On the Blind Equalization of Continuous Phase Modulated Signals Using the Constant Modulus Criterion, IEEE Transactions on Signal Process, 2007, Vol.55 (3) 1047-1061.

[5] N.Deshpande, Fast Recovery Equalization Techniques for DTV Signals, IEEE Transactions on Broadcasting, 1997, Vol.43 (4) 370-377. 